\title{
Big Data Skills Sustainable Development in Healthcare and Pharmaceuticals
}

\author{
António Pesqueira ${ }^{1} \cdot$ Maria José Sousa ${ }^{1}$ (D) - Álvaro Rocha ${ }^{1}$
}

Received: 12 September 2020 / Accepted: 30 September 2020 / Published online: 9 October 2020

(C) Springer Science+Business Media, LLC, part of Springer Nature 2020

\begin{abstract}
Big Data technology is one of the most promising organizational processes within the Healthcare and Pharmaceutical industry and crucial for any company that wants to preserve the competitive advantage in the market, where most of the organizational structures are already struggling with the right skills and knowledge to fully support existing business needs for storing and processing and even analyzing information. This paper aims to examine the extent to which new Big Data technology and datarelated processes are developing different professionals skills and competencies within the Healthcare and Pharmaceutical industries, and creating sustainable development in addressing critical organizational challenges in recruiting, retaining, and discover professional skills that can fully support the advances and exponential growth of Big Data technology benefits. This research paper also highlights the significant aspects of Big Data in professional technical and process oriented skills development, and the influence it has on organizational business processes including how various internal functions will need to adapt to new circumstances with renewed competency and skills development programs for departments that are strongly connected to the business and analytical needs. We conducted a focus group with twenty-five industry based professionals' ranges from analysts to executive directors to better assess the necessary knowledge to answer the proposed research questions: (1) which professional skills can big data influence in employee development and (2) how can organizations adapt their employee skills to big data. Regarding the key research limitations/implications most of the article and research was built on the foundation of the literature review and the performed focus group. The conceptual recommendations and observations presented provide solid empirical evidence but should be subjected to more comprehensive, large-scale empirical testing and validation. It's recommended for future research a more extensive sample of companies, organizations, and interviewees. Studying a broader set of similar research questions in more homogeneous organizations could provide deeper insights into the process, governance, and stakeholder dimensions of Big Data within specific contexts. Therefore this study contributes to explore in-depth and systematically to what extent Big Data technology and processes are currently influencing the healthcare and pharmaceuticals industries where to the best of the authors' knowledge, it is the first focus group dealing with the presented research questions.
\end{abstract}

Keywords Big data $\cdot$ Skills development $\cdot$ Healthcare and pharmaceutical $\cdot$ Professionals skills

\section{Introduction}

Healthcare and Pharmaceutical companies, independently of the company size, are extracting information from different sources and with the main objective to apply the knowledge of the collected data into a better and more informed decision

Maria José Sousa

mjdcsousa@gmail.com; maria.jose.sousa@iscte-iul.pt

1 Department: Management, ISCTE-Instituto Universitario de Lisboa, Avenida das Forças Armadas Lisboa, Lisboa, Portugal support processes and reveal meaningful business insights and data outputs to scrutinize business models and business approaches.

The Big Data trend within the Healthcare and Pharmaceutical the industry is helping different organizations from different therapeutical areas, markets, and regions to reach up to decisions within the stipulated timeframe, and uncover real-world insights from payers, competitors, patients, regulators, etc.

The innovative transformation in different functions like sales, marketing, medical affairs, regulatory or market access is already a new pressing reality with increased importance from the pandemic status for the industry after COVID-19. 
Ubiquitous big data management infrastructures and advanced analytical technologies started to be integral roles in competitive and digital organizations nowadays, where the Healthcare and Pharmaceutical industry is not an exception to that [42].

On the Healthcare and Pharmaceutical level, for instance, the biggest corporations have been identifying for some year's now new areas to foster big data and data science transformation in several different internal departments, like Research \& Development (R\&D), Sales, Marketing, and Regulatory Affairs.

The aims of the big data transformation policies and processes are to strengthen business and technical processes but as well to create new organizational capabilities to seize opportunities and innovations that can advance data management $\&$ discovery technologies enable for organizations and individuals.

Rapid digital evolution and new digital innovations after the pandemic crisis (COVID-19) have also challenged the status quo of the industry and the way the data is treated, analyzed, and discovered.

Big Data skills are seen as a paradigm shift and as a contrast of traditional data analytics methods and big data technology and processes may generate systemically, disruptive impacts, so this paper also considers the disruptiveness of big data strategies within organizations' business models, value creation, and value networks.

Hence Big data services and solutions have become integral parts of advanced companies and analytical solutions are increasingly being embedded in organizations' daily operations and activities, so the phenomenon of big data has been perceived as a paradigm shift and a powerful business weapon to also the Healthcare and Pharmaceutical industry [47].

The current and future data transformation is recognized to be multifaceted, complex, and uncertain [44]. It can have influences on organizations' resources, capabilities, processes, structures, skills, and investment needs, for example. Furthermore, it has been found that organizations 'big data transformation affects their cultures, skills maturity, business insights, value creation, business models, and stakeholders in their value networks.

In order to better understand all described key concepts and challenges this paper consider the phenomenon of big data from a skills development perspective and examines the interplay of big data, employee skills development, and organizations in order to extend the knowledge of big data role for skills development and to provide a systematic approach to conducting big data in organizations with the right employee's skills.

By identifying key elements relevant to the big data thematic research and understand how to systematically carry out specific skills development we are then able to fully understand the importance of big data technologies within the selected industry and gain a better comprehension of all the several implications for decision-making processes in different organizations settings.

The key objectives of this research paper are to examine the extent to which new Big Data technology and data-related processes are developing different professionals skills and competencies within the selected industries, and highlight the significant aspects of professional technical and processoriented skills development, and the influence it has on connected processes including how various internal functions will need to adapt to new circumstances with renewed competency and skills development programs.

To identify relevant articles dealing with big data skills in the selected professionals' group and the skills required to perform analytics and manage big data-related processes, we used a vast range of search terms in conjunction with "big data" and "healthcare" or "pharmaceuticals" or "skill", and successfully we were able to bring a practical overview and application of the potential results for this research with a solid background development and theoretical review.

Therefore this study contributes to explore in-depth and systematically to what extent Big Data technology and processes are currently influencing the healthcare and pharmaceuticals industries where to the best of the authors' knowledge, it is the first focus group dealing with the presented research questions.

\section{Development of professional skills}

According to literature, in nowadays Big Data professionals across different industries should possess a range of different skills, where these skills include not only well-established topics such as data quality management and data warehousing, but also emerging topics such as data science and distributed file systems.

This growing need for big data specialists with analytical capabilities is also increasing tremendously in healthcare and pharmaceuticals as we will see in the next sections.

The imperative of professional skills development as a resource and competence in Healthcare and Pharmaceutical organizations has been progressively noticed. These professionals can demonstrate critically and unique capabilities to develop novel and pioneering improvements in the way organizations are implementing and developing big data projects, thus achieving greater organizational effectiveness.

The Healthcare and Pharmaceutical professional skills for big data projects are determined by the tasks that are required in their work environment [45], and as the complexity of big data processes and related technology increases, the skills of Healthcare and Pharmaceutical professionals must also increase.

Hence technology and processes relevant skills, including programming, data visualization, and management expertise, 
and the software management field is essential for professionals to take on big data roles and focus on innovation that can bring all necessary outcome and data insights applied to the Healthcare and Pharmaceutical context (e.g. drugs development, clinical research, etc.).

Yet, the challenges of big data implementation faced by Healthcare and Pharmaceutical, professionals go beyond the typical IT or technical problems. Specifically, in addition to the expected skills that require a high degree of business understanding, statistics, and advanced analytics, there are now more demanding job requirements, such as business functionality and data science skills, especially in an increasingly changing business and technological environment [2].

Therefore, professionals involved directly or indirectly with Big Data need a variety of skills for successful practice and successful execution of the necessary activities. Although much focus has been placed on data management or analytics, it is becoming increasingly recognized that a range of professional skills are required for safe and effective practice and better project management [12]. These skills set might include communication, reflection, ethical practice, teamwork and professional development.

\section{Big data in healthcare}

Big Data as a business concept is growing relevance and importance in several different industries where Healthcare is no exception.

This concept has been a growing hype term in several different discussions and projects planning, where some of them can be attributed to the promotional initiatives by companies like IBM, IQVIA, Truven Health and other leading technology companies who have been investing significantly in building new analytical capacity for healthcare institutions (e.g. hospital, transplant centers, etc.).

Big data topics and definitions have been evolving rapidly, with some confusion and misunderstanding in regard to data science, data warehousing or traditional data management processes and techniques [18].

All kinds of data can be examined using Big Data. This data can include data such as diagnoses, vital signs, biomarkers data, prescribed medicines, and several types of reported symptoms and diseases that can be examined using Big Data processes. In healthcare management, prescription records trial data is in two forms i.e., digital/electronic and paper. Data can be in any form like Electronic Health Records (EHR) of a patient, clinical reports, doctor's prescription, diagnostic reports, and images, etc. HER Data can be evaluated in different ways where it can include blood pressure readings, height, weight and glucose measurements, etc.

Also from a research laboratories perspective and medical universities, data mining and big data has been used to predict models for diabetes, oncology and rare diseases. Currently, several researchers have revealed that a patient's record can be studied using data analytics to foresee the future risk of metabolic syndrome as well as oncology or other complex diseases in patients who currently do not have any symptoms.

The value of Big Data in healthcare is a big opportunity to develop patient-driven outcomes, sustainable models of healthcare delivery and ultimately to maximize the potential of scientific and clinical developments.

But unfortunately, healthcare systems are struggling to spend their investments where it has the highest impact and Big Data might help these systems to allocate spending where it really makes a difference.

However, there is a risk that overly restrictive data privacy laws and consent requirements to Healthcare Professionals (HCP) and patient's data could affect healthcare ability to realize the potential of Big Data for research, diagnosis, treatments, etc. [27].

Big Data strategies in healthcare are being effective to better inform decision making in generating already high savings in different system components, by optimizing innovation, improving the efficiency of research and clinical diagnosis, and building new tools for physicians, consumers, insurers, governmental payers and regulators to meet the promise of personalized and more specific treatment approaches.

This opportunity is driving Healthcare providers and insurance companies in complex business environments experiencing a growing trend in the types and volumes of available data.

The healthcare data growth is generated from several sources, including the R\&D process itself, retailers, patients, and caregivers.

\section{Big data in pharmaceuticals}

In the Pharmaceutical sector, advanced big data infrastructures, and technologies have enabled the emergence of new digital platforms and business intelligence ecosystems with new business models, customer experiences, and value creation possibilities. Companies such as Roche, Novartis, and AstraZeneca are examples of companies that is being able to operate in big data environments and collect advanced insights into critical operations like clinical trials, R\&D investigations, commercial profiling, and segmentation, and overall decision support processes.

These companies, along with other life sciences and healthcare, players have drastically changed the way the industry is consuming its data and redesign business models, for example, inpatient engagement, HCPs targeting, and clinical research activities.

The uses for Big Data in Pharma are limitless. Clinical, Commercial and Market Access teams can all benefit from Big Data insights. Some key activities like understand and assess HCPs and Key Opinion Leaders (KOLs) needs, develop better promotional targeting, gather Patient-Related 
Outcomes (PROs), explore speaker programs or congresses opportunities to further contextualize disease and drug response through continuous medical education and develop better products promotionally messaging or market segmentation strategies are some examples of activities benefiting already from big data internal services.

Part of taking advantage of Big Data involves making data capture as electronic as possible, and several pharma companies are increasingly using advanced analytical of capturing and transmitting commercial and medical team's responses by using large data sets and complex computational algorithms like machine learning or predictive analytics.

Recently FDA and EMA (also other governmental regulatory agencies) approved different wearable devices for use as a medical complement and used to record patient data (also in support with patient support programs). These kinds of approvals are clear examples of how pharmaceutical patient engagement platforms are being used to collect data quickly and more reliably from the patient. These and similar devices will improve data capture and increase datasets to yield more reliable results.

As a fact Big Data application vary slightly among pharmaceutical companies and consultants (e.g. IQVIA, Truven).

Hence pharma companies are more likely to focus Big Data initiatives on current products to grant the success of drugs already on the market, where consultancy companies are more likely to contribute to the development of new technologies and solutions. Overall, the prevalence of these broad Big Data-driven market intelligence initiatives will continue to increase, as data become more useable and the benefits more obvious in pharma.

\section{Big data 3 V's}

Data main definitions fall into three major areas of operations and strategy. According to literature, big data analysts should possess a range of complex skills in addition to huge amounts of data (Volume), data processing speed needs (Velocity) and the various forms of data resources to collect structured and unstructured data of all types (Variety).

The first major area refers to the volume, where the magnitude of data, .data sizes are reported in multiple terabytes and petabytes. The most relevant definitions of big data volumes are relative and vary by factors, like the time and the type of data. What may the reality of volume today may not be the same reality in the future, where mainly the storage capacities will exponentially increase, allowing even bigger data sets to be captured [29].

The second area refers to variety refers, where the structural heterogeneity in a dataset will help us to better understand this point. With all technological signs of progress allowing pharma companies to use different types of structured, semi-structured, and unstructured data, the way companies, and business functions are able to derive results and insights tabular data found in spreadsheets or relational databases, text, images, audio, and video are examples of all complexity of big data variety.

Thus Healthcare and Pharmaceutical organizations have been hoarding unstructured data from internal sources (e.g., patients' data, clinical trials, adverse events) and external sources (e.g., HCPs behavioral data, sales data like inpatient and outpatient). However, the emergence of new data management technologies and analytics in the Healthcare and Pharmaceutical industry, which can enable organizations to leverage different sets of data in their business processes (e.g. market access, sales, marketing), is exactly the area that is meant to big data. For instance, natural language processing or predictive analytics technologies will continue to increase and to be empowered in this industry where Healthcare and Pharmaceutical manufacturers are keen to acquire intelligence about patients, key opinion leaders, and healthcare professionals and their journeys and preferences. This invaluable information is leveraged in decisions related to product launches, supply chain management, and segmentation [24].

However the usage of big data analytics in pharma, even small and medium-sized enterprises (SMEs) in the biotechnology, space is still not capable to handle with massive volumes of semi-structured data to improve business models designs and implement effective sales and marketing strategies and personalized patient and customer's recommendation systems.

The last major area of big data refers to the velocity, in terms of the rate at which data are generated and the speed at which it should be analyzed and processed.

The proliferation of digital devices and augmented reality in medical prescriptions, diagnosis, treatment support systems, patient support programs and much more has led to an unprecedented rate of data creation and is driving a growing need for real-time analytics and real-world evidence-based decision making.

This data provides sound information about patients, such as preferences, needs, treatment adherence, demographics, and drug reaction patterns, which can be analyzed in realtime to create real patient value.

Given the soaring the popularity of smartphones, Healthcare and Pharmaceutical, and biotechnology manufacturers have to deal with hundreds of thousands of clinical and medical data sources that demand real-time capacity to understand results and variations in terms of diagnostics, treatment, and medical records. Traditional data management systems are not capable of handling huge data feeds instantaneously through medical programs or clinical trials research activities and this is where big data technologies come into play. Big Data is offering infinite possibilities that can enable pharma companies to create real-time intelligence from high volumes of 'perishable' data.

Thus we still have a very narrow interpretation of what that Big Data means to pharma and we lose track of the fact that there are multiple steps to the data analysis pipeline, whether 
the data is considered as being small or big. Different companies are also struggling with data acquisition, data governance and defining solid data transfers workflows that bring data quality and excellence in terms of insights generation capacity.

\section{Organizational dimensions and elements}

It has been argued that organizations should have a strategic focus on how to carry out long-term big data projects within themselves.

Traditionally, organizations have developed data management strategies to manage data infrastructures, tools, applications, and services $[13,20]$ that support organizations' functions and processes [39].

Differentiated from traditional data management strategy, notions of Big Data business strategy is used to refer to organizations' strategic focus on how to implement advanced Big Data technologies and transformation within themselves and their value networks.

In that manner Healthcare and Pharmaceutical business and Big Data transformation strategies reflect businesses' perspectives on data management and consider the technical and human resources and capabilities required to digitalize organizations' structures, products, services, processes, and business models. Traditional data management and Big Data strategies take into account how organizations create and deliver differential value for vital stakeholders such as customers and patients in their value networks.

To simplify the concepts of big data, the notion of the concept is applied in this article to describe the data management of large data sets from pharma organizations and key elements needed to consider when managing human resources and related professionals in their structures and activities. A Big Data skill thus is a holistic view of specific skills Healthcare and Pharmaceutical professionals adopt to evaluate, manage, and govern the big data journey [28].

Several different authors over time have identified some external triggering events that push the organization to transform their internal business skills and activities into big data forms. Changes in Big Data consumption, analytics and demands propel organizations to digitalize internal processes and develop systems and technologies that enhance business decision processes experiences (Tables 1, 2, 3 and 4).

\section{Research approach and methodology}

In this chapter, an overview of the research approach and methodology is presented. This research investigates big data as a potential for Healthcare and Pharmaceutical professionals' skills development and research's main scope is providing a systematic approach and focus group for carrying out big data and deploying novel skills in organizations' activities connected with Big Data.

The research is positioned as investigating Healthcare and Pharmaceutical organizations and their aim is to increase knowledge and understanding of the elements that influence how novel skills are being management deployed in Healthcare and Pharmaceutical and biotechnology organizations and their value in managing and orchestrating big data programs.

\section{Research objectives}

Considering the challenges presented in section 1.1, the pervasiveness of big data transformation and the organizational dimensions and elements, this article is positioned to explore big data potential in professional skills development in Healthcare and Pharmaceutical organizations. This paper is aimed at developing a systematic approach and focus group to big data as skills development through the following research objectives:

- RO1. Which professional skills can big data influence in employee development?

- RO2. How can organizations adapt their employee skills to big data?

In that manner the aim of RO1 is to identify the skills that big data influence. $\mathrm{RO} 2$ investigates the ways in which organizations systematically address and carry out big data projects in adaptation to their employee skills. Both research objectives are intended, first, to improve Healthcare and Pharmaceutical organizations' ability to effectively adopt and implement novel big data technologies with the right individual skills and, second, to help Healthcare and Pharmaceutical organizations achieve the set objectives of having the right employee skills in adopting new big data projects and technologies. This paper is intended to achieve these research objectives, as well as present the prior theoretical literature and better understand individual skills sets with Healthcare and Pharmaceutical professionals through a focus group.

\section{Focus group}

Based on the literature review, we developed ten focus group questions and invited several healthcare and pharmaceutical professionals to help us in better understanding the relevant professional's skills that were relevant to be analyzed and understood the impact of Big Data.

During the focus group discussion, we first asked questions about the participants' experiences with big data and opinions regarding big data skills for healthcare and pharmaceuticals professionals without showing our list of questions. 
Table 1 organizational elements connected with Big Data Strategies

\begin{tabular}{ll}
\hline Organizational elements & Authors \\
\hline $\begin{array}{l}\text { Change management and data } \\
\text { governance }\end{array}$ & Chanias [5]; Haffke et al. [17]; Tabrizi et al. [38]; Wulf et al. [46] \\
Culture and skills management & Alfaro et al. [1]; Chanias [5]; Dery et al. [10]; Eden et al. [11]; Morakanyane \\
& et al. [26]; Singh and Hess [37]; Tabrizi et al. [38]; \\
Big Data & Sadowski [32]; Pink et al. [30], Christin [8]; Seaver [35]; Schrock, Shaffer \\
& [34] \\
Alfaro et al. [1]; Berman and Marshall [3]; Davenport and Westerman [9]; & Goul [15]; Gust et al. [16]; Legner et al. [23]; Ross et al. [31]; Sahu et al. \\
& [33]; Sebastian et al. [36]; Tabrizi et al. [38]; Westerman, \\
& Alfaro et al. [1]; Chanias [5]; Chanias and Hess [6, 7]; Dery et al. [10]; Eden \\
& et al. [11]; Bharadwaj et al. [4]; Gust et al. [16]; Legner et al. [23]; Matt \\
Leadership and top & et al. [25]; Sebastian et al. [36]; Singh and Hess [37]; Sebastian et al. [36]; \\
management involvement & Tabrizi et al. [38]; Tumbas et al. [40]; Wulf et al. [46] \\
& Alfaro et al. [1]; Chanias [5]; Chanias and Hess [6, 7]; Haffke et al. [17]; \\
& Weill and Woerner [43]; Wulf et al. [46] \\
Alfaro et al. [1]; Chanias [5]; Davenport and Westerman [9]; Eden et al. [11]; & Henriette et al. [19]; Kane et al. [21]; Kutzner et al. [22]; Legner et al. [23]; \\
indicators & Matt et al. [25]; Morakanyane et al. [26]; Sahu et al. [33]; Tumbas et al. \\
Process & [40] \\
Alfaro et al. [1]; Berman and Marshall [3]; Chanias [5]; Davenport and \\
Westerman [9]; Dery et al. [10]; Eden et al. [11]; El Sawy et al. (2016); \\
Gimpel et al. [14]; Gust et al. [16]; Haffke et al. [17]; Henriette et al. [19]; \\
Hess et al. [20]; Kane et al. [21]; Legner et al. [23]; Ross et al. [31]; \\
Sebastian et al. [36]; Singh and Hess [37]; Tabrizi et al. [38]; Tumbas et al. \\
[40]; Weill and Woerner [43]; Wulf et al. [46] \\
\hline Skills and capabilities & \\
\hline &
\end{tabular}

Table 2 Focus group questions

\section{Focus Group Questions}

Added Value of Big Data to Healthcare and Pharma: Strategies to increase the awareness of the added value of Big Data

Skills Development: How to strengthen human capital with respect to the increasing need for a workforce that can utilize the potential of Big Data

Data Sources: Explore sources of Big Data in Pharma and how to secure their quality and safety

Open Data and Data Sharing: Promote open use and sharing of Big Data in pharma without compromising patients' and healthcare professionals (HCP) rights to privacy and confidentiality

Applications and Purposes: Increase target-oriented application of Big Data analysis in pharma based on the needs and interests of stakeholders including patients

Data Analysis: Identify the potentials of Big Data analysis, improve analytical methods and facilitate the use of new and innovative analytical methods

Governance of Data Access and Use: Governance mechanisms to ensure secure and fair access and use of Big Data for research

Standards: Existing standards for Big Data to enhance and simplify its application and improve interoperability

Financial Resources: Forms of investment to warrant cost-effectiveness and sustainability

Legal Aspects and Privacy Regulations: Legal and privacy regulation of Big Data impacting the selected industry
Two participants mentioned some initial concerns with big data: "We are still struggling to deal with big data in practice"; "to analyze huge data amounts (...) and benefit from our professional skills"; "although modern databases promise high performance, performance optimization is still an issue".

To that, we started to understand that basic database knowledge was already presented as a very important skill set. Another participant highlighted without seeing any of the ten questions that "once you start integrating data from different sources, you need more available skills and better IT processes".

After showing our preliminary questions to the participants, they generally agreed on our identified questions but argued in favor of concentrating on skills relevant to the industry.

\section{Focus groups methodology}

The sampling methodology used identified relevant stakeholders who were a representation of Healthcare and Pharmaceutical professional performing directly or indirectly the work and other key informants to assure comprehensive representation of the Big Data core functions with regard to the field to compare the "gap" between the current state and anticipate future state skills desired and required.

The Focus group participants were selected from the following stakeholder categories: 
Table 3 Top-ranked categories, relevant topics and key words

Focus Group Main Categories $\quad$ Most Relevant Discussed Topics $\quad$ Key Words and Frequency (more than 5)

Questions

\begin{tabular}{|c|c|}
\hline $\begin{array}{l}\text { Added Value } \\
\text { of Big Data } \\
\text { to } \\
\text { Healthcare } \\
\text { and Pharma }\end{array}$ & $\begin{array}{l}\text { - When utilized correctly, big data gives } \\
\text { healthcare companies the information } \\
\text { needed to streamline customer service } \\
\text { processes that personalize healthcare and } \\
\text { create best practices for working with } \\
\text { consumers or patients. } \\
\text { - Customers can receive a more thorough } \\
\text { and personalized experience. } \\
\text { - Improvement of efficiencies for } \\
\text { operational management of business } \\
\text { models and use cases }\end{array}$ \\
\hline
\end{tabular}

Skills Domain Knowledge: Decision Making,

Developme- Healthcare and Pharmaceutical, Patient

nt and/or HCPs Data Analysis Understanding, Product/Compliance Data Analysis, Data Privacy and Legal, Business Models and Industry specific processes knowledge

- Big Data Relevant Skills: Data Visualization, Prescriptive Analytics, Data mining, data processing and governance

- IT Skills: Software Engineering, Programming, Data quality management, distributed file systems, Cloud Computing

Data Sources - Data Sources and types: clinical, claims, market and competitors, tenders, patient, sentiment from HCPs and patients, cost activity, sales (units, vials, blisters, packages), $R \& D$, supply chain, patients generated, medical images.

- New Data sources: smartphones, payer records, patient portals, generic and public databases, EHRs, Wearable devices

- Others: clinical trials, insurance payments, EMRs, images, Dx, Rx, Tx, fitness records and history, ADR, observational data, market research, telemedicine

Open Data and - Components and servicesNetwork and

Data

Sharing portalsDiagnostics - Data mining and analysis to identify causes of illness

- Preventative medicine - leveraging analytics and data analysis of genetic, lifestyle and social circumstances to prevent diseases

- Medical research - data driven medical and pharmacological research to cure disease and discover new treatment and medicines

Applications and

- Analytical Techniques: modelling, simulation, machine learning, visualization, data mining, statistics, web mining, optimization, text mining, forecasting and social network analysis

- Prevention, Diagnosis, Treatment, Homecare
- Identification of relevant information entities

- Automated decision of relevant structures and data types

- Standards fostering algorithm integration and development

- Patient journeys and treatment pathways understandings were also referred towards development of technical skills in staff with big data management potential and identified as a new opportunity with benefits to the entire organization.

- Healthcare and Pharmaceutical understanding, supply chain management, market access, pharma products commercialization, clinical trials data and HCPs management.

- Technical skills needs for pharmaceutical staff focused on big data and skills-based topics like bioinformatics are drugs and molecules data, adverse events, pharmacovigilance events, regulations, general supply chain management functions and basic microbiology or Healthcare and Healthcare and Pharmaceuticals techniques data analysis.

Creation of mature data

modelsImprovement of existing data standards (e.g. biomedical)

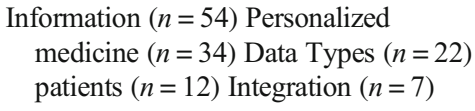

Information $(n=54)$ Personalized medicine $(n=34)$ Data Types $(n=22)$ patients $(n=12)$ Integration $(n=7)$

Decision making ( $n=32)$ Data analysis $(n=24)$ Knowledge $(n=17)$ Programming $(n=15)$ Data quality $(n=11)$ Cloud computing $(n=11)$ Systems integration $(n=8)$ Data mining $(n=6)$

trials $(n=17)$ Prescriptions $(n=7)$ smartphones $(\mathrm{n}=5)$ patients $(n=5)$ Integration $(\mathrm{n}=5)$
- Semantic data representation Context and Protocols $(n=15)$ Hospital dossiers forecasting models representation

- Data usage procedures and relevant access profiles $(n=13)$ Formularies $(\mathrm{n}=12)$ patients

$(n=12)$ Public data $(\mathrm{n}=7)$
- Research improvements

- Technological improvements

- Reporting and evaluation

- Monitoring and prediction/simulation
Analytics $(n=21)$ Forecasting $(n=14)$ Data Types $(\mathrm{n}=22)$ Business outcomes $(\mathrm{n}=12)$ Insights $(\mathrm{n}=7)$ 
Table 3 (continued)

Focus Group Main Categories

Most Relevant Discussed Topics

Key Words and Frequency (more than 5)

Questions

- Enable patients to manage their own

prevention, ensure diagnosis with

personalized care pathways, enable more

effective therapies

Data Analysis • Reduction of adverse medication events - • Research and development - pharmaceuharnessing of big data to spot medication tical errors and flag potential adverse reactions - Drug repurposing

- Cost reduction: identification of value that $\cdot$ Health record guided drug development drives better patient outcome for $\quad$ Next generation sequencing long-term savings - Personalized Healthcare

- Population health - monitor big data to - Treatment Adherence - Compliance identify disease trends and health strate- - Adverse events detection gies based on demographics, geography $\bullet$ Patient pre-profiling and socio economics - Influencer Profiling

Governance of • Standardization • Drug Discovery

Data Access • Heterogeneity - Medical Imaging

and Use - Interactions - Track and prevent diseases

- Longitudinal follow ups - Predictive analytics

- Linkage

- Genomics

Standards - Domain context

- Domain context - Unstructured data integration

- Predictive modelling

- Develop scale algorithms

- Security best practises for non-relational data stores

- Computing platforms

- Automation

- Granular Audits

- Risk modelling

- Customer segmentation

- Recommendation engines and models

- Real-time predictive analyticspatient lifetime value

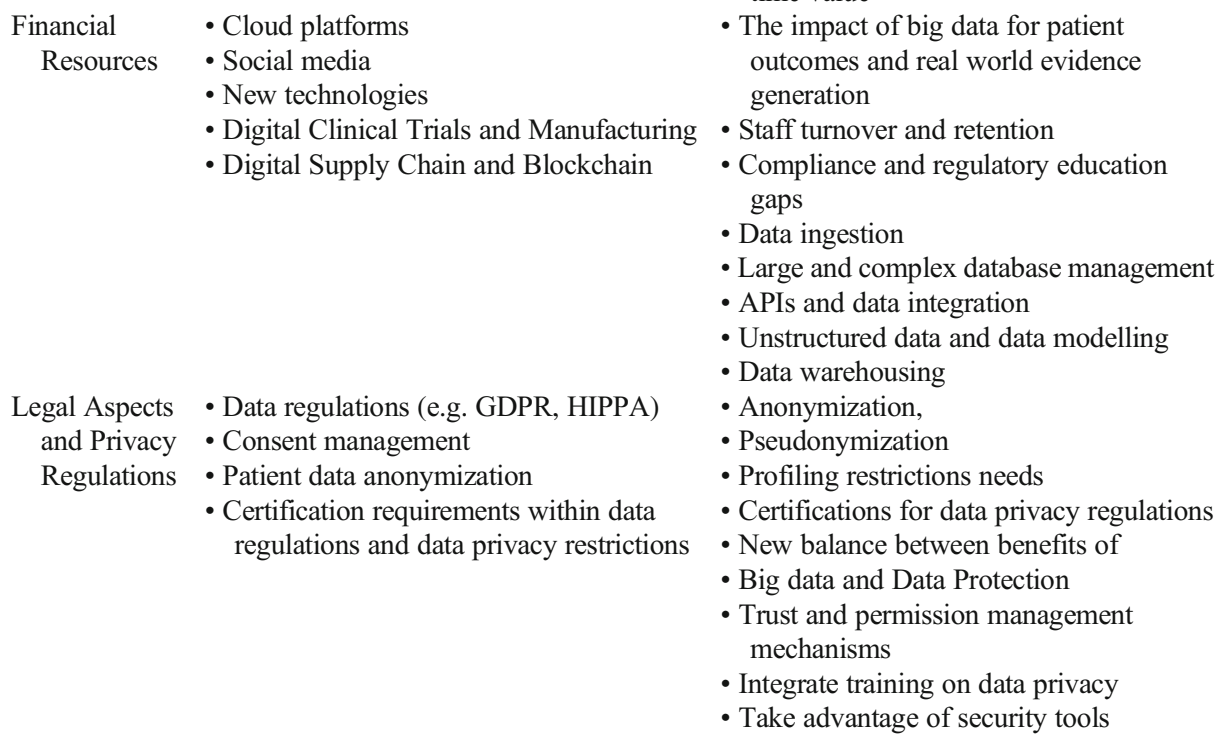

Adverse Events $(\mathrm{n}=34)$ Patient $(n=31)$

Analytics $(\mathrm{n}=21)$ Strategies $(\mathrm{n}=11)$

Outcomes $(n=10)$ Insights $(n=8)$

Governance $(n=24)$ Data usage $(n=17)$

Processes $(n=12)$ Matches $(n=6)$
APIs $(n=9)$ Technologies $(n=7$ Costs $(\mathrm{n}=5)$

Automation ( $\mathrm{n}=13)$ Risks $(n=9)$

Procedures $(n=6)$ SOPs $(n=6)$
Privacy $(n=24)$ Consent $(n=22)$ Data protection $(\mathrm{n}=21)$ Anonymization $(\mathrm{n}=15)$ Trust $(\mathrm{n}=12)$ Training $(\mathrm{n}=7)$ Skills $(n=6)$

- Commercial and Marketing Business Analysts or Directors

- IT staff or analytics management professionals

- Digital professionals' part of IT or Marketing departments

- Commercial and Marketing Operations Analysts and Business Leaders

- Local affiliates sales and marketing directors
The initial invitation to participate was sent to 39 invitee's part of three different Healthcare and Pharmaceutical companies. Twenty-five participants accepted our research invitation to share their opinions and experiences in remote focus group discussions. The entire group of twenty-five professionals was divided into smaller groups of 5 individuals each for better 
Table 4 Summary of identified skills

\begin{tabular}{|c|c|}
\hline \multirow[t]{7}{*}{ Domain Knowledge } & Decision Making \\
\hline & Healthcare and Pharmaceutical \\
\hline & Patient and/or HCPs Data Analysis Understanding \\
\hline & Product/Compliance Data Analysis \\
\hline & Data Privacy and Legal \\
\hline & Business Models \\
\hline & Industry specific processes knowledge \\
\hline \multirow{6}{*}{$\begin{array}{l}\text { Big Data Relevant } \\
\text { Skills }\end{array}$} & Data Visualization \\
\hline & Prescriptive Analytics \\
\hline & Data mining \\
\hline & Data Processing and Governance \\
\hline & Descriptive Analytics \\
\hline & Data Science Understanding \\
\hline \multirow[t]{9}{*}{ IT Skills } & Software Engineering / Programming \\
\hline & Data Quality Management \\
\hline & Distributed File Systems \\
\hline & Systems Architecture and Integration \\
\hline & Web/Cloud Computing \\
\hline & Databases Management \\
\hline & Data Warehousing \\
\hline & Data Security \\
\hline & Stream Processing \\
\hline \multirow[t]{4}{*}{ Others } & Business Modelling Improvement \\
\hline & Business development \\
\hline & Project Management \\
\hline & Scorecards and Dashboards \\
\hline
\end{tabular}

facilitation and management of all discussions and interview questions.

Participants represented a wide diversity of positions including:

- Senior Directors, Directors, and Associate Directors

- Managers

- Business analysts

- Technical or IT specialists

We conducted in a total of ten sessions using the zoom technology starting from June 5th, 2020 to June 22nd, 2020, sorting participants into smaller groups with similar titles and responsibilities. The sessions were 60-90 $\mathrm{min}$ in length and PowerPoint slides were used to guide the interviews and facilitate the sessions.

Statistically, local affiliates sales and marketing directors and commercial and marketing operations analysts and business leaders accounted for 54\% of the participants, followed by commercial and marketing business analysts or director and digital professionals part of IT or marketing at 32\%, and IT staff or analytics management professionals at $12 \%$.
Participants were located in different geographical regions, like Switzerland, United States, Brazil and Canada.

Open-ended questions were used to gain the best perspective and scope of individual responses and what mattered to the individual's area of interest and expertise. Facilitator notes ensured uniformity among focus group discussions and kept each discussion on target. Participants introduced themselves including name, organization and position. The purpose of the focus group, as per email communication, was repeated as a reminder. An opportunity was given to rescind consent to participate. Responses were not recorded, and participants were assured their comments would be kept confidential. Ground rules were established and used during each discussion (Fig. 1).

Each question was read twice to the participants, and the facilitator encouraged full engagement from each participant to maximize a thorough compilation of data.

If any answers were unclear, the facilitator would ask probing questions, such as:

- Can you talk about that some more?

- Help me understand what you mean.

- Can you give me an example?

The facilitator summarized main points of participant responses for a question before moving on to the next question. After the last question, the facilitator read each question one more time to give participants an opportunity to make additional comments.

\section{Focus groups analysis process}

Part of the focus group strategy we defined three main phases (planning for analysis, information collection and management and interpretation), where each of those phases had related steps.

This process had the main intention to produce quality and removing any bias from all collected information and analysis, and ultimately produce the best focus group outputs as possible.

\section{Focus group questions}

We developed a list of ten questions to guide the discussion of each focus group session. Each session used the same group of questions. Participants received the questions in advance of their scheduled session to encourage advance preparation. Since the composition of each group varied by job duties and titles, several perspectives were represented in their responses. 
Fig. 1 Stakeholder categories and number of participants

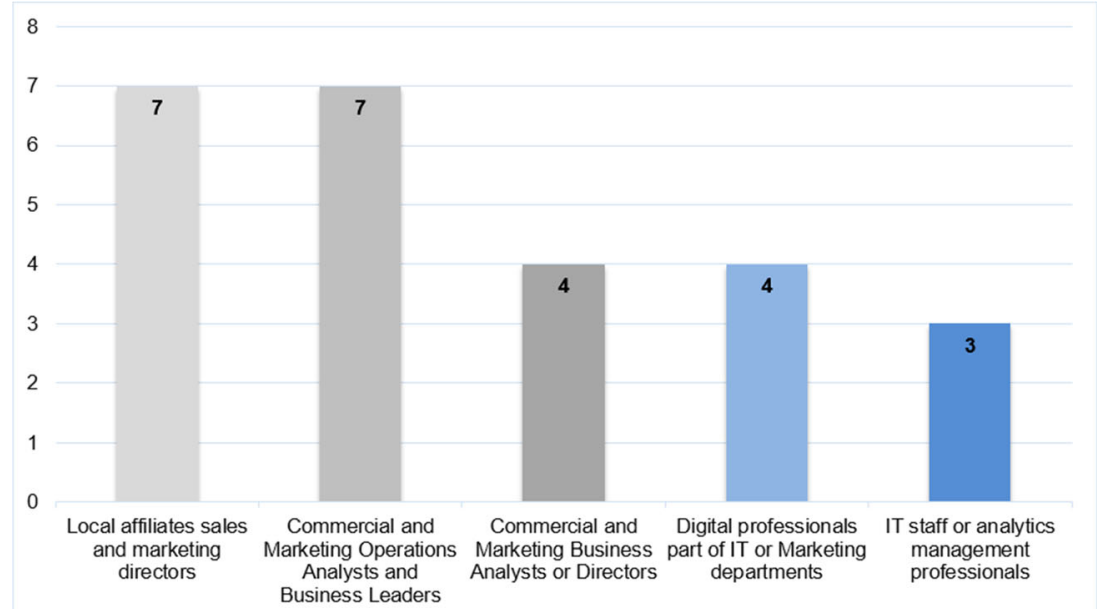

\section{Focus group results and process conclusions}

Across all groups, participants' responses regarding their most critical answers fell into prominent categories, that according to the process presented on Fig. 2 from the previous section point 2.4.2., we created a summary overview with all main key terms and answers highlights for a more effective and simple understanding of all results.

The following table lists the top topics (out of 177) to each of the presented questions and mentioned most often during focus group discussions. A complete list of topics generated from participant responses are on a separate excel file from all the collected notes and used for python analysis of natural language processing (NLP).

The below presented key words and frequency are only displaying the words and/or terms that were mentioned more than 5 times for a question of visualization optimization of the results and reading optimization for the reader.

Results of the focus group analysis revealed the above major findings. This section aims to provide a generic overview of the key highlight and takeaways to the most pressing topics.

Several different internal departments find it challenging to hire appropriately educated staff in big data-related topics, to motivate staff, and once trained, to retain skilled staff.
Fig. 2 Focus group analysis process

\section{Planning for analysis}

Step 1: Define the research and analysis groups from collect text

Step 2: Choosing a recording method

Step 3 . Targeting the analysis

Step 4: Developing the focus group questions

Step 5: Budgeting time, objectives and resources

\section{Information collection and management}

Step 1: Review Zoom recorded sessions and note-taking

Step 2: Debriefing with the focus group and follow up questions

Step 3: Natural Language Processing analysis for key terms capture and words frequency

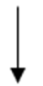

\section{Interpretation}

Step 1: Variables identification and influence terms analysis

Stcp 2: Considcring the context and relcvant arcas

Step 3: Looking at all findings with a "industry-based lens"

Step 4: Drawing conclusions and making final recommendations

Step 5: Validating the results and cross-checking for similarities with literature review analysis 
We can extract several insights from the added value of big data to the industry and skills development, where it's important to highlight that most of the relevant topics were pointing into the need of real-time information, new healthcare trends like precision medicine, data types and importance of big data to decision support processes.

From the focus group results, we also need to highlight the major relevance of topics like data sources, applications and purposes from analytics to business processes and less important processes connected with legal and regulatory requirements and internal policies.

\section{Results discussion}

The consensus of the focus groups was that big data is still not fully present in the organizations that all involved professionals were part of, but there was a general consensus that big data will be an integral part of different departments to assure the necessary capacity and capability to meet the needs for analytics and insights generation. Participants were extremely motivated to share their experiences - both positive and challenging. The findings, which were the thoughts of the group identified that big data priorities are dependent on the specific business needs and could not prioritize clearly specific skills from professional in terms of support and management of big data processes.

The focus group data show that domain expertise and data governance are vital skills for big data teams and professionals within Healthcare and Pharmaceutical. Priority domain expertise topics include Healthcare and Pharmaceutical understanding, supply chain management, market access, pharma products commercialization, clinical trials data and HCPs management.

Technical skills need for pharmaceutical staff focused on big data and skills-based topics like bioinformatics are drugs and molecules data, adverse events, pharmacovigilance events, regulations, general supply chain management functions and basic microbiology or data analysis techniques. Patient journeys and treatment pathways understanding were also referred towards development of technical skills in staff with big data management potential and identified as a new opportunity with benefits to the entire organization.

Throughout the focus groups, participants noted a list of shared interests and concerns which included:

- The impact of big data for patient outcomes and realworld evidence ${ }^{1}$ generation

- Staff turnover and retention

- Certification requirements within data regulations and data privacy restrictions

\footnotetext{
${ }^{1}$ Real World Evidence (RWE) is an ideal tool to perform a proof of concept that a pharmaceutical company deems necessary to demonstrate the real scenario of healthcare in practice.
}

- Compliance and regulatory education gaps

- Salary competition with other entities

\section{Summary of findings and contributions for research objectives}

The results from the literature review and the focus group highlighted several skills relevant to answer the first research question: Which professional skills can big data influence in employee development?

Below is a summary of those skills and according all collected data and information.

In terms of the major findings to the second research questions the data suggested that IT development and use within organizations are processual and contextual and that business involvement should be a major goal is to have impacts on Big Data practice.

Another fundamental conclusion from the focus group data was the main goal of organizations that would like to adapt their employee skills to big data, the research data presents that skills from professionals that are advanced in information systems should be effectively deployed in organizations through programs of internal innovation and centers of excellence, where topics like big data are the center of the activities focus and the investment is made in full transparency and under strict and modern governance.

Traditionally organizations from healthcare and pharmaceuticals have a good response rate in terms of accepting theories from relevant disciplines such as IT governance, economics, and business intelligence but they still fail in terms of accepting and thriving in implementation of practical frameworks applied in complex data environments contexts. The Big Data phenomenon in all methodology steps provided a clear set of answers based on the conclusions and data analysis from the literature review and focus group, where the main observations allow us to conclude that organizations that systematically and effectively implement novel digital technologies into their activities are the ones that gain more competitive advantage and better-added value from Big Data. Consequently, traditional approaches or business models are still struggling to understand the phenomenon of Big Data and digital transformation and for that reason, they might not enough innovation and business development perspectives. Expanding knowledge about these factors that influence and are essential for Big Data is a major need for healthcare and pharmaceuticals.

\section{Conclusions}

\section{Practical implications}

This article provides several practical implications for organizations' decision-makers and managers. First, management 
needs to have a strategic approach to creating a culture and capabilities for tracking novel big data skills to better empower their employees and develop better competencies development framework.

Second, management should have systematic procedures for the design and implementation of big data standards, resources, and data privacy procedures. Third, an iterative, lean approach to experimenting with novel digital technologies, preferably from external business institutions increases organizations' opportunities to learn about new big data skills and how to align them to organizations' own structures and processes. Additionally, systematic technology experiments enable developing and renewing contemporary value propositions and business models in existing and new markets. Alternatively, strategic alliances and collaboration with digital start-up ecosystems may provide environments for learning and extend knowledge about Big Data innovations.

\section{Limitations}

Most of the article and research was built on the foundation of the synthesis of the literature and the performed focus group. The conceptual recommendations and observations presented provide solid empirical evidence but should be subjected to more comprehensive, large-scale empirical testing and validation. Another limitation is the relatively small sample of companies, organizations, and interviewees. Studying a broader set of similar research questions in more homogeneous organizations could provide deeper insights into the process, governance, and stakeholder dimensions of Big Data within specific contexts.

Thus, a more extensive empirical research area could enable observing and comparing the obstacles and barriers that prevent skills development from Big Data in a specific research context.

\section{Motivation for further studies}

This paper presented an overview of the challenges faced in moving to a future where Big Data technologies and processes will require new collaborative, and multidisciplinary skills development processes and moving key skills and process areas identified in the literature review and focus group conclusions into a center stage. In this paper, we also presented key factors and areas from the focus group discussions, which we see Big Data as a key influencer in bringing data, environmental, and business analysts into a common and coherent environment where businesses and organizational processes can thrive. In such a space, they are able to work together with new skills to utilize their different expertise in order to champion data science and Big Data solutions to some of the environmental business challenges. In addition to highlighting the significant progress already made in the development and application of Big Data in the healthcare and pharmaceutical space, this paper also sets out a clear and defined research roadmap on how we feel is the way forward in this rapidly emerging business domain. We believe this can form the focal point for an international research community progressing the cultural and business changes for open and collaborative discussions in a necessary framework for new professionals skills development, ongoing case studies of good practice, and infrastructural and methodological developments required to enable Big Data to support a significant increase in innovative and new industry business capabilities.

During the focus group discussions, it was further emphasized the need for healthcare and pharma organizations to bring these skills, and enthusiasm for practicing them, to new work environments and needs.

\section{Future recommendations}

Big Data is not a trivial concept and many historical events show how new technologies and innovations have changed the course of many industries and even disrupted businesses. As an emerging research phenomenon, therefore, Big Data as a potential skills development factor provides several alternative paths in the research field. Considering organizational perspectives, the theory of the resource-based view of the industry could consider organizations' strategies, resources, and competitive advantages [41], whereas organizational culture could investigate levels such as artifacts, values, and underlying assumptions.

Exploring the organization's Big Data maturity and skills asymmetry might provide new perspectives for the organization's Big Data influence in the professional's skills development.

Due to the rapid evolution and diffusion of big data technologies and processes in global markets, future research could also investigate the skills development and business models in more broaden contexts.

A large-scale curriculum or skills development framework mapping exercise would be useful to identify how healthcare and pharma professional frame the objectives, teaching and learning methods, and assessment of professional skills.

Hence organizations may find it difficult to assess the disruptiveness of rapidly changing technologies and processes in their daily activities and planning. The theory of disruptive innovation and Big Data presented in this article can also provide perspectives for assessing technology discontinuities and disruptiveness. However, further research developing more unified theoretical foundations to address the disruptive elements of Big Data and Data Science innovations and technologies and their impacts on organizations' diverse dimensions might also be fruitful for researchers in the Healthcare and Pharmaceuticals industry. 
Further research is warranted into the learning methods that can be offered by Big Data programs to develop professional skills, along with the investigation into the drivers and barriers to skill development during careers.

\section{Final conclusions}

In this study, there is a hybrid attempt to effectively answer the presented research questions using analysis and context understanding from literature review and a focus group with twenty-five industry experts and professionals.

We identified 4 major summary areas of identified required skills from Big Data and applied to the industry, 10 top-ranked categories, relevant topics, and key words and 5 major shared interests and concerns from the focus group discussions:

1. The impact of big data for patient outcomes and realworld evidence generation

2. Staff turnover and retention

3. Certification requirements within data regulations and data privacy restrictions

4. Compliance and regulatory education gaps

5. Salary competition with other entities

Moreover, this methodology approach was implemented also to gain some light in terms of key factors and influence areas from Big Data to professionals' skills development in healthcare and pharmaceuticals.

The proposed methodology approach considers that professional competencies maybe acquired after a Big Data project is implemented, but the period between the project inception and skill attainment is likely to be undervalued and the impact on working factors from existing big data projects. Most of the times only after starting the implementation of a big data project, we realize the lack of skills or knowledge from the working teams, and as highlighted in some of the focus group discussions.

In that manner, it would be reasonable to assume that healthcare and pharmaceutical professionals also need to possess these Big Data relevant attributes to better contribute to the success of related activities and investments connected with new technology implementation and data management and decision support related processes.

Further conclusions from the focus group were also highlighted in terms of the many causes of professional skill deficits in several different organizations, which will require further assistance in the contextualization and development of these competencies during and after any Big Data project.

The target skills need to be identified and integrated into their employees' experience and learning activities, assessment and appraisal processes from early onboarding training and into their career maturity.
Consequently, it could be perceived from the focus group meetings that the group enriched the discussions, aggregating information that went beyond those necessary to perform the task, improving reflections and contributing to the involved people's training.

In this sense, the adopted two research methods permitted associating Big Data potential with the skills development of healthcare and pharma professionals, with several conclusions and recommendations for the future. The group itself manifested increased awareness of reality. In addition, the discussions and explanations from the researchers made it possible to generate descriptive and more critical knowledge of the situation.

In conclusion, the potential of Big Data within the Healthcare and Pharmaceutical also serves to increase the skills development of the industry professionals and allow internally functions to be better prepared in terms of data management and technology expertise.

The main purpose of this article was to demonstrate and communicate the value of big data in enhancing the knowledge and skills of professionals and the better understanding the associated processes and technology influence from Big Data. All the described research questions were validated and several conclusions were drawn in terms of possible next steps and research questions.

We were able also to conclude that big data services and solutions have become integral parts of advanced companies and analytical solutions are increasingly being embedded in organizations' daily operations and activities.

Resulted from the focus group discussions, we were able to understand how the domain knowledge around key processes like decision making, data privacy, business models and much others are directly connected with big data and skills development from healthcare and pharmaceuticals professionals.

We were also able to describe all relevant skills in big data contexts and adapted to the selected industries and all relevant IT skills like software engineering and programming, data quality and database management, and others.

The key objectives of this research paper were discussed and analyzed and a better understanding created of the extent to which new Big Data technology and data-related processes are developing different professionals' skills and competencies and influencing the selected industries in new processes design and business development activities.

Funding This study was funded by Fundação para a Ciência e Tecnologia, Grant: UIDB/00315/2020.

\section{Compliance with ethical standards}

Conflict of interest The authors declare that they have no conflict of interest.

Ethical approval All procedures performed in studies involving human participants were in accordance with the ethical standards of the 
institutional and/or national research committee and with the 1964 Helsinki declaration and its later amendments or comparable ethical standards.

Informed consent Informed consent was obtained from all individual participants included in the study.

\section{References}

1. Alfaro, E., Bressan, M., Girardin, F., Murillo, J., Someh, I., \& Wixom, B. H., 2019, BBVA's Data Monetization Journey. MIS Q. Exec. 18 (2), 4, 2019. 5, 2019.

2. Bassellier, G. V. and Benbasat, I, Business Competence of Information Technology Professionals: Conceptual Development and Influence on IT-Business Partnerships. MIS Quarterly, 28, 4, 673-694. This article contains supporting information online at Google Scholar 2004.

3. Berman, S., \& Marshall, A., 2014, The next digital transformation: from an individual-entered to an everyone-to-everyone economy. Strategy \& Leadership, 42, 5, 9-17.

4. Bharadwaj, A., El Sawy, O., Pavlou, P., and Venkatraman, N., 2013., Digital business strategy: toward a next generation of insights. MIS Quarterly, 37, 2, 471-482., 2013.

5. Chanias, S., Mastering digital transformation: the path of a financial services provider towards a digital transformation strategy. In Proceedings of the 25th European Conference on Information Systems, ECIS, Guimarães, Portugal, pp. 16-31 2017

6. Chanias, S., \& Hess, T., 2016a, How digital are we? Maturity models for the assessment of a company's status in the digital transformation. Management Report/Institute für Wirtschaftsinformatik und Neue Medien, 2, 1-14.

7. Chanias, S., \& Hess, T., 2016b, Understanding digital transformation strategy formation: insights from Europe's automotive industry. In PACIS, 2016, p. 296,

8. Christin, A. 2017, Algorithms in practice: Comparing web journalism and criminal justice, First Published July 16, 2017 Research Article https://doi.org/10.1177/2053951717718855

9. Davenport, T. H., \& Westerman, G., 2018, Why so many highprofile digital transformations fail. Harvard Business Review.

10. Dery, K.; Sebastian, I. M. \& van der Meulen, N., 2017, The Digital Workplace Is Key to Digital Innovation. MIS Quarterly Executive, $16,2,, 135-152$.

11. Eden, R., Jones, A. B., Casey, V., \& Draheim, M., 2019, Digital transformation requires workforce transformation. MIS Quarterly Executive, 18, 1, 1-17.

12. Gamble, A., Observing and predicting the 2015/16 El NiñoBulletin of the journals.ametsoc.org 2017

13. Gerster, D., Digital Transformation and IT: Current State of Research., PACIS, 2017 - aisel.aisnet.org, retrieved from https:// aisel.aisnet.org $/ \mathrm{cgi} / \mathrm{viewcontent}$.cgi? article $=1150 \&$ context $=$ pacis2017 2017

14. Gimpel, H., Hosseini, S., Huber, R., Probst, L., Röglinger, M., \& Faisst, U., 2018,

15. Goul, M., 2018, Poised Between 'a Wild West of Predictive Analytics' and 'an Analytics of Things Westworld Frontier', MIS Q. Exec., 17, 4, 333-347. 76

16. Gust, G., Flath, C.M., Brandt, T., Strohle, P., \& Neumann, D., 2017, How a traditional company seeded new analytics capabilities. MIS Quarterly Executive, 16, 3, 215-230.

17. Haffke, I., Kalgovas, B., \& Benlian, A., 2017, Options for Transforming the IT Function Using Bimodal IT. MIS quarterly Executive, 16, 3, 215-230.
18. Hand, P., Global guarantees for enforcing deep generative priors by empirical risk, Conference On Learning Theory, proceedings.mlr.press accessed on June 21st. 20202018.

19. Henriette, E., Feki, M., \& Boughzala, I., Digital transformation challenges. In MCIS Proceedings, p. 33 2016,

20. Hess, T., Matt, C., Benlian, A., \& Wiesböck, F., 2016, Options for formulating a digital transformation strategy. MIS Quarterly Executive, 15, 2, 123-139.

21. Kane, G. C., Palmer, D., Phillips, A. N., Kiron, D., \& Buckley, N., 2015, Strategy, not technology, drives digital transformation. MIT Sloan Management Review, 14, 1-25.

22. Kutzner, K., Schoormann, T., \& Knackstedt, R., Digital transformation in information systems research: a taxonomy-based approach to structure the field. In Proceedings of the 26th European Conference on Information Systems, ECIS, 2018, p. 56, Portsmouth, UK 2018.

23. Legner, C., Eymann, T., Hess, T., Matt, C., Böhmann, T., Drews, P., \& Ahlemann, F., 2017, Digitalization: opportunity and challenge for the business and information systems engineering community. Business \& Information Systems Engineering, 59, 4, 301308.

24. Mamoshina, P., Ojomoko, L., Yanovich, Y., Ostrovski, A., Converging blockchain and next-generation artificial intelligence technologies to decentralize and accelerate biomedical research and healthcareOncotarget, from ncbi.nlm.nih.gov 2018

25. Matt, C., Hess, T., \& Benlian, A., 2015, Digital transformation strategies. Business \& Information Systems Engineering, 57, 5, 339-343.

26. Morakanyane, R., Grace, A. A., \& O'Reilly, P., Conceptualizing digital transformation in business organizations: a systematic review of literature 2017.

27. Nowak, J., Raj, S., Gertz, S., and Grunwell, J., Improved risk stratification in pediatric septic shock using both protein and mRNA biomarkers. PERSEVERE-XPAmerican journal of atsjournals.org, accessed on June 22nd, 2020, 2017.

28. Oussous, A., Benjelloun, A., Lahcen, A., Big Data technologies: A surveyJournal of King Saudia Arabia from Elsevier.com 2018

29. Pappas, I., Krogstie, J., Giannakos, M., Big data analytics capabilities: a systematic literature review and research agendaInformation Systems from Springer, accessed from https://doi.org/10.1007/ s10257-017-0362-y 2018

30. Pink, S., Sumartojo, S., Lupton, D., Bond, C., Mundane data: The routines, contingencies and accomplishments of digital living, First Published March 27, 2017 Research Article https://doi.org/10. 1177/2053951717700924 2017

31. Ross, J. W., Beath, C. M., \& Sebastian, I. M., 2017, How to develop a great digital strategy. MIT Sloan Management Review, 58, 2, 7-9.

32. Sadowski, J., When data is capital: Datafication, accumulation, and extraction, First Published January 7, 2019 Research Articl https:// doi.org/10.1177/2053951718820549 2019

33. Sahu, N., Deng, H., \& Mollah, A., Investigating the critical success factors of digital transformation for improving customer experience. IN CONFIRM 2018 Proceedings, 18. Pp.1-13 2018.

34. Schrock, A., Shaffer, G., Data ideologies of an interested public: A study of grassroots open government data intermediaries, Big Data \& Society, journals.sagepub.com 2017

35. Seaver, N., Algorithms as culture: Some tactics for the ethnography of algorithmic systems, Big Data \& Society, journals.sagepub.com 2017

36. Sebastian, I. M., Ross, J. W., Beath, C., Mocker, M., Moloney, K. G., \& Fonstad, N. O., 2017, How big old companies navigate digital transformation. MIS Quarterly Executive 16, 3, 197-213.

37. Singh, A., \& Hess, T., 2017, How chief digital officers promote the digital transformation of their companies. MIS Quarterly Executive, 16, 1, 1-17. 
38. Tabrizi, B., Lam, E., Gerard, K., \& Irvin, V., 2019, Digital transformation is not about technology. Harvard Business Review.

39. Teubner, T., 2013, Probes of the small $\mathrm{x}$ gluon via exclusive $\mathrm{J} / \psi$ and $\Upsilon$ production at HERA and the LHCJournal of High Energy from Springer, accessed on July 23rd, 2020.

40. Tumbas, S., Berente, N. \& vom Brocke, J. , 2017, three Types of Chief Digital Officers and the Reasons Organizations Adopt the Role, MIS Quarterly Executive, 16, 2, , 121-134.

41. Wade, M., Hulland, J., The resource-based view and information systems research: Review, extension, and suggestions for future research, MIS quarterly, from dl.acm.org, accessed on June 15th, 20202004.

42. Wang, Y., Kung, L., Byrd, T., Big data analytics: Understanding its capabilities and potential benefits for healthcare organizations, Technological Forecasting and Social Change, accessed from Elsevier.com 2018

43. Weill, P., \& Woerner, S. L., 2015, Thriving in an increasingly digital ecosystem. MIT Sloan Management Review, 56, 4, 27-34.
44. Williams, P., Woodward, A., Cybersecurity vulnerabilities in medical devices: a complex environment and multifaceted problem, PMID: 26229513Published online 2015 Jul 20. doi: https://doi. org/10.2147/MDER.S50048 2015

45. Wu, S., Joseph, A., Hammonds, A., Celniker, S., Yu, B., and Frise, E., Stability-driven nonnegative matrix factorization to interpret spatial gene expression and build local gene networks, first published April 6, 2016. https://doi.org/10.1073/pnas.1521171113.

46. Wulf, J.; Mettler, T. \& Brenner, W. , 2017, Using a Digital Services Capability Model to Assess Readiness for the Digital Consumer, MIS Quarterly Executive, 16, 3, 171-195.

47. Zhang, C., Jiang, B., Li, M., Zhu, Y., and Zhang, P., The lysosomal $\mathrm{v}$-ATPase-Ragulator complex is a common activator for AMPK and mTORC1, acting as a switch between catabolism and anabolism. Cell metabolism, 2014 - Elsevier, 2014.

Publisher's note Springer Nature remains neutral with regard to jurisdictional claims in published maps and institutional affiliations. 